\title{
What Influences Trust in Survey Results? Evidence from a Vignette Experiment
}

Sven Stadtmüller

Henning Silber

Christoph Beuthner

GESIS - Leibniz Institute for the Social Sciences

A revised version of this manuscript was published online first on July 29, 2022 in the International Journal of Public Opinion Research, available at: https://doi.org/10.1093/ijpor/edac012 


\begin{abstract}
Today, there are more survey results available than ever before. This increase in survey data is, however, accompanied by a decline in survey quality. Thus, it is more likely than in the past that citizens and politicians get a biased picture of public opinion when relying on survey results. Those misperceptions can have worrying consequences for political discourse and decision-making. With the present study, we aim to investigate to what extent the public draws on survey quality information when evaluating the trustworthiness of a survey result. To explore this research question, we implemented a vignette experiment in an online panel survey $(n=3,313)$ in which each respondent was confronted with four different, randomly assigned descriptions of a survey and then asked to evaluate the trustworthiness of the respective survey result. The survey descriptions varied regarding the methodological information provided (i.e., sample size, sampling method, and sample balance). The results showed that survey quality information only had a minor effect on the perceptions of trust compared to respondents' characteristics, such as pre-existing opinions on the topic or general trust in science. Yet, trust in the survey result was significantly influenced by the sample size and sample balance, but not by the sampling method. Finally, in line with information processing theory, the relevance of survey quality information increases with the cognitive abilities of the respondent.
\end{abstract}

\title{
Keywords
}

survey reports, survey data quality, sample size, representativeness, sampling method, cognitive abilities, information processing, heuristics 


\section{Introduction}

Survey results on topics of political interest have an important role in the democratic process (Verba, 1996). Not only do they reflect public opinion and may thus serve politicians as a guiding principle for policy-making (Gallup and Rae, 1940; Brodie et al., 2001; Newport et al., 2013). For citizens, survey results are also a source of political information and allow them to compare their viewpoints with the ones from their compatriots. In this regard, there is substantial evidence that polling results exert a reciprocal effect on individual attitudes as well as on public opinion and political discourse (Moy and Rinke, 2012; Searles et al., 2018).

Today, there are more survey results available than ever before (Groves, 2011; Leeper, 2019). The main reason for that is the rise of web surveys since the early 2000s (Couper and Miller, 2008). Through tailored software solutions and commercial access panel providers, it has become relatively easy and inexpensive to collect survey data (Baker et al., 2010). However, there is evidence that this quantitative increase in survey data is accompanied by a decline in survey quality (Greszki et al., 2015; Gummer et al., 2018). Most online surveys, for instance, use convenience samples, which usually do not allow the generalization to a wider population (Clifford et al., 2015; Mercer et al., 2017; Yeager et al., 2011). The higher prevalence of survey data of questionable quality increases the likelihood that politicians and citizens get a biased picture of public opinion when relying on survey results, and this is worrying from a democracy theory point of view. As a result, initiatives have gained in importance that aim at fostering transparency regarding the methodology of surveys. Professional associations such as the American Association of Public Opinion Research (AAPOR), for instance, have published detailed guidelines of what should be reported and shared when survey results are published (AAPOR, 2021). However, the media rarely complies with those standards (Meyer, 1990; Miller and Hurd, 1982), and even many scientists fail to do so when reporting survey results (Audibert et al., 2020; Hermanni and Lemcke, 2017). 
Yet, even in situations in which a survey's methodology is fully disclosed, one should not be overly optimistic that recipients primarily rely on this information when evaluating the trustworthiness of a survey result. This is because previous research has demonstrated that polling results, like any other messages communicated through the mass media, are prone to processing biases. More specifically, in an effort to avoid cognitive dissonances, recipients are more likely to reject a survey result that contradicts with their pre-existing attitudes, while they are more inclined to accept results that fit to their views (Tsfati, 2001; Kuru et al., 2017; Madson and Hillygus, 2020). In the field of Political Psychology, these information processing biases are known as the congruency and discongruency bias and range under the joint umbrella of motivated reasoning (Kunda, 1990; Lodge and Taber, 2000; Redlawsk, 2002). When people engage in motivated reasoning, they process incoming information with the primary objective to maintain their belief system. Thus, if messages contradict with their beliefs, recipients engage in reasoning against the incoming content. However, the engagement in motivated reasoning has its limits, and people also strive, to varying degrees, for accuracy in their judgments and evaluations. In line with this, research has found information about the quality of the survey to affect the perceived credibility of its results (Salwen, 1987) and methodological disclosure to moderate motivational biases in credibility assessments (Kuru et al., 2017).

What is not clear from previous research, however, is the relative importance of both factors, information on survey quality and motivated reasoning, on the perceived trustworthiness of a survey result. Recent studies from Kuru et al. (2017, 2020) suggest motivated reasoning to play a more important role with respect to survey trust evaluations than methodological details. However, with the 2016 presidential election, gun control, and abortion, the authors focused on issues on which a large majority of citizens is likely to hold clear and strong opinions. In such instances, people are more likely to engage in motivated 
reasoning, and this may, in turn, result in an overestimation of its general importance (Taber and Lodge 2006; Taber, Cann, and Kucsova 2009).

In our study, we use a vignette experiment (Auspurg and Hinz, 2015), in which each respondent received multiple randomly assigned survey descriptions with varying information on survey quality. For each description, respondents were asked to assess the trustworthiness of the survey result. This experimental design enabled us to disentangle the relative importance of survey quality information and respondents' characteristics, such as preexisting opinions or general trust in science. Thus, our first research question is:

RQ1: What is the relative importance of survey quality information for the perceived trustworthiness of a survey result?

Apart from the relative importance of survey quality information for evaluating the trustworthiness of a survey result, it is an open question which specific quality indicators the public relies on when assessing the quality of a survey and ascribing trust to its result. Survey experts use a variety of quality criteria (Sturgis et al., 2016) such as the sampling method, the response rate, and the sample balance. However, due to their limited knowledge about surveys, the public probably has to rely on heuristics (Tversky and Kahneman, 1974) to evaluate the quality of a survey. More specifically, one might expect people to focus on heuristics for survey quality that are most accessible and intuitive to them. To this end, our second research question is:

RQ2: Which survey quality indicators does the public rely on when assessing the trustworthiness of a survey result?

Finally, the relative importance of survey quality information as well as the effects of survey quality indicators on the evaluation of trust may vary between recipients with different levels of cognitive ability. In models of information processing and persuasion, cognitive abilities are a prerequisite to comprehend incoming messages and to process them more thoroughly (Chaiken, 1980; Petty and Cacioppo, 1986). This suggests that recipients with 
high cognitive abilities may pay more attention to differences in survey quality. Further, one might expect people with high cognitive abilities to be more familiar with survey quality indicators due to their higher levels of exposure to political media and since they spent more time in educational institutions. Hence, our third research question is:

RQ3: Does a respondent's cognitive ability moderate the effects of survey quality indicators on the perceived trustworthiness of a survey result?

\section{Method}

\section{Data}

Data for this study came from a commercial online access panel and was collected using the survey programming and management software Questback Unipark. ${ }^{1}$ To ensure a diverse sample of the German population, quotas with respect to age (six levels), education (three levels), and sex (two levels) were implemented when inviting the panel members. Since the main topic of the survey was data sharing behavior, respondents were also screened based on their Twitter usage and the manufacturer of their smartphone (Apple or Samsung). The survey was fielded between November $28^{\text {th }}$ and December $17^{\text {th }}, 2019$. Selected panel members received an email from the panel provider that included a link to the survey. From the 26,339 invitations, a total of 10,484 respondents saw the starting page of the survey (participation rate: $39.8 \%$ ); 6,863 panel members who did not fulfill the quota assignment were screened out (Screen-out rate: 65.5\%). Another 485 respondents did not complete the survey (completion rate: $86.6 \%$ ) but were considered for our analytic sample if they had valid values on all relevant variables. In sum, the analytic sample comprises 3,313 participants. The median response time of the survey was 12.4 minutes and the target vignette experiment on survey quality information and trust evaluations was placed at the beginning of the survey.

\footnotetext{
${ }^{1}$ In commercial online access panels, respondents sign up to be invited to surveys on a regular basis. For their participation, they receive small monetary incentives, usually in the form of panel points.
} 


\section{Vignette Experiment}

In vignette experiments, survey respondents receive multiple short descriptions of hypothetical objects or situations (the vignettes) and are asked to evaluate them with respect to a certain outcome variable. The vignettes differ in several attributes (dimensions) with each dimension consisting of at least two levels (Auspurg and Hinz, 2015; Mutz, 2011).

In our study, the vignettes were brief descriptions of a survey dealing with the question of whether or not commuters should benefit from higher tax allowances due to rising fuel prices (see Table 1 for an example). The survey descriptions varied in five dimensions with mostly two levels (see Table 1), resulting in a $2 \times 2 \times 2 \times 2 \times 3$ factorial design with 48 unique vignettes.

Table 1. Dimensions and levels of the vignettes

\begin{tabular}{ll}
\hline Dimension & Levels \\
\hline \multirow{2}{*}{ Source / Survey sponsor } & The German Automobile Association \\
& The German Institute of Transport Research \\
Survey result & 36 percent favour higher tax allowances for commuters \\
& 72 percent favour higher tax allowances for commuters \\
Representativeness & Not mentioned \\
& Mentioned \\
Sample size & 100 respondents \\
& 1,000 respondents \\
& 10,000 respondents \\
Sampling method & Not mentioned \\
\end{tabular}

Example for a vignette (the dimensions and their respective levels are written in italics):

The German Automobile Association recently presented the results of a representative survey. Accordingly, 36 percent of the Germans favour higher tax allowances for commuters due to rising fuel prices. For the survey, 1,000 randomly selected people were questioned.

How much do you trust in this survey result? [1 "not at all" to 7 "completely"]

The dimensions representativeness, sample size, and sampling method provided respondents with information about the quality of the survey and are regarded as important quality indicators by survey statisticians (Sturgis et al., 2016). For our vignettes, we decided to include the adjective "representative" to provide information on sample balance. Although this term is vague even among survey statisticians, in Germany it is frequently used by the 
news media when polling results are reported. In that context, "representative" mainly aims at indicating that the respondents constitute a reduced image of the entire population, and may serve as an information shortcut (i.e., a heuristic) for a balanced net sample. As a second indicator, we included the net sample size with three levels (100, 1,000, and 10,000 respondents). Finally, the criterion of a probability-based survey was included in the vignettes as a random selection of the respondents. Apart from these quality indicators, the dimensions source and survey result provided additional details about the survey.

Each respondent was assigned to four different vignettes. While for four of the five dimensions the levels were drawn and combined completely at random, we initially randomly allocated each respondent to one of two decks with 24 vignettes each. These vignettes shared the same survey result. This meant that for each respondent, the survey result was randomly fixed to either 36 or 72 percent for all four vignettes. We did this to dampen the effects of motivated reasoning due to varying results in the vignettes while drawing the respondents' attention to the survey characteristics to uncover the maximum potential of survey quality information on the assessment of trust in the survey result.

After each vignette, respondents were asked to rate their level of trust in the result of the survey on a seven-point scale ranging from (1) "not at all" to (7) "completely" trustworthy.

\section{Other Measures}

For analyzing RQ3, we relied on formal education as a measure for cognitive abilities. We created a variable representing respondents with low, medium, or high cognitive abilities based on their reported school-leaving graduation. ${ }^{2}$ We additionally included age (in years),

\footnotetext{
${ }^{2}$ Respondents without a graduation or with lower secondary education (Hauptschulabschluss or Polytechnische Oberschule ( $8^{\text {th }}$ or $9^{\text {th }}$ grade) in the former German Democratic Republic) were assigned to the group with low cognitive ability, while respondents with upper secondary education (Realschulabschluss or Polytechnische Oberschule ( $10^{\text {th }}$ grade) in the former German Democratic Republic) were assigned to the group with medium cognitive ability. Finally, respondents with higher secondary education (Abitur, Fachabitur or Erweiterte
} 
sex (with females coded 1), and a measure on general trust in science (measured with a fivepoint rating scale from "do not trust at all" to "fully trust") to avoid confounding the effects of cognitive abilities with other related measures and to check whether the effects of the survey quality indicators changed when these control variables were included in the statistical models.

\section{Analyses}

In total, 13,189 vignettes were evaluated. However, these evaluations were not independent from another since they were distributed over 3,313 respondents. To account for this hierarchical data structure, we estimated multi-level regression models (Hox et al., 2017) with evaluations nested in respondents.

In multi-level models, the overall variance of the dependent variable can be split up into variance on the different analytical levels (Rabe-Hesketh and Skrondal, 2012). Accordingly, our starting point of analysis was the null model without covariates to estimate the share of the overall variance that was due to the differences in the observations for the same respondents. In essence, the higher this share, the more important were the differences in the survey descriptions and hence the more important for the respondents was information on survey quality when evaluating trust in the survey results. ${ }^{3}$ In contrast, the higher the share of variance on the respondent level, the closer the link between the reported trust and characteristics of the respondents, irrespective of differences in the survey descriptions (RQ1).

In a second step, we added the vignette dimensions to the multi-level model to analyze which survey quality indicators respondents primarily relied on when ascribing trust to the

Oberschule in the former German Democratic Republic) belong to the group of respondents with high cognitive ability.

${ }^{3}$ Variance on the level of the survey descriptions also includes variance that is due to differences in the source but does not include differences in the survey result since this is a between-subject variable. 
survey results (RQ2). Since each vignette was evaluated approximately 275 times $^{4}$, it was possible to disentangle the influence of each dimension on respondents' evaluation. We also estimated the same models together with demographics and general trust in science as control variables. To investigate whether there are combined effects of the survey quality indicators on the trust evaluations, we also included all two-way interactions for the three quality indicators and also integrated a two-way interaction for the other two dimensions, source and survey result.

For analyzing RQ3, we carried out the same analytical steps as for RQ2 but estimated separate models for each of the three groups of formal education to examine the impact of different levels of cognitive ability. Those models did not include interaction terms for reasons of statistical power.

\section{Results}

The first column in Table 2 displays the results of the null model. Accordingly, respondents' characteristics accounted for the majority of the overall variance of the dependent variable, trust in the survey result (62.9\%). In contrast, the share of the overall variance between observations of the same evaluators was $37.1 \%$. Regarding the first research question, this suggests that rather attributes of the evaluators than attributes of the survey dominate when people ascribe trust to a survey result.

\footnotetext{
${ }^{4}$ This was calculated by dividing $\mathrm{n}(3,313)$ by the number of vignettes $(48)$, multiplied with the number of vignettes per respondent (4).
} 
Table 2. Results of the vignette experiment on the trustworthiness of survey results (full sample)

\begin{tabular}{|c|c|c|c|c|c|c|c|c|c|c|c|c|}
\hline & \multicolumn{3}{|c|}{ Null model } & \multicolumn{3}{|c|}{$\begin{array}{l}\text { Model with } \\
\text { vignette } \\
\text { dimensions } \\
\end{array}$} & \multicolumn{3}{|c|}{$\begin{array}{l}\text { Model with } \\
\text { vignette } \\
\text { dimensions } \\
\text { and controls }\end{array}$} & \multicolumn{3}{|c|}{$\begin{array}{c}\text { Model with } \\
\text { vignette dimen- } \\
\text { sions, controls and } \\
\text { interactions }\end{array}$} \\
\hline & $\mathrm{b}$ & se & $\mathrm{p}$ & $\mathrm{b}$ & se & $\mathrm{p}$ & $\mathrm{b}$ & se & $\mathrm{p}$ & $\mathrm{b}$ & se & $\mathrm{p}$ \\
\hline \multicolumn{13}{|l|}{ Fixed Effects } \\
\hline $\begin{array}{l}\text { Constant } \\
\text { Vignette dimensions }\end{array}$ & 4.53 & .023 & $* * *$ & 4.30 & .038 & $* * *$ & 3.00 & .111 & $* * *$ & 2.99 & .114 & $* * *$ \\
\hline \multicolumn{13}{|l|}{ Source / Survey sponsor } \\
\hline \multicolumn{13}{|l|}{ Survey Result } \\
\hline \multicolumn{13}{|l|}{ Representativeness } \\
\hline Mentioned & & & & .107 & .018 & $* * *$ & .108 & .018 & $* * *$ & .183 & .036 & $* * *$ \\
\hline \multicolumn{13}{|l|}{ Sample size } \\
\hline 1,000 respondents & & & & .242 & .022 & $* * *$ & .243 & .022 & $* * *$ & .289 & .038 & $* * *$ \\
\hline 10,000 respondents & & & & .439 & .022 & $* * *$ & .439 & .022 & $* * *$ & .468 & .038 & $* * *$ \\
\hline \multicolumn{13}{|l|}{ Sampling Method } \\
\hline Probability-based selection & & & & -.001 & .018 & & .001 & .018 & & .001 & .036 & \\
\hline \multicolumn{13}{|l|}{ Controls } \\
\hline Age (in years) & & & & & & & .011 & .002 & $* * *$ & .011 & .002 & $* * *$ \\
\hline Female respondents & & & & & & & -.052 & .046 & & -.052 & .046 & \\
\hline Trust in science & & & & & & & .235 & .018 & $* * *$ & .234 & .018 & $* * *$ \\
\hline \multicolumn{13}{|l|}{ Two-way interactions } \\
\hline Representative $\mathrm{x} 1,000$ & & & & & & & & & & -.101 & .044 & $*$ \\
\hline Representative $\mathrm{x} 10,000$ & & & & & & & & & & -.087 & .044 & $*$ \\
\hline Probability x 1,000 & & & & & & & & & & .009 & .044 & \\
\hline Probability x 10,000 & & & & & & & & & & .027 & .044 & \\
\hline Representative $\mathrm{x}$ Probability & & & & & & & & & & -.026 & .036 & \\
\hline Research Institute x 72 percent & & & & & & & & & & .090 & .036 & $*$ \\
\hline \multicolumn{13}{|l|}{ Variances of Random Effects } \\
\hline Variance: Constant & \multicolumn{3}{|c|}{$1.54(.044)$} & \multicolumn{3}{|c|}{$1.55(.044)$} & \multicolumn{3}{|c|}{$1.45(.041)$} & \multicolumn{3}{|c|}{$1.45(.041)$} \\
\hline Variance: Residual & \multicolumn{3}{|c|}{$.909(.013)$} & \multicolumn{3}{|c|}{$.870(.012)$} & \multicolumn{3}{|c|}{$.870(.012)$} & \multicolumn{3}{|c|}{$.869(.012)$} \\
\hline Proportion of Level 2-Variance & \multicolumn{3}{|c|}{$62.9 \%$} & \multicolumn{3}{|c|}{$64.1 \%$} & \multicolumn{3}{|c|}{$62.4 \%$} & \multicolumn{3}{|c|}{$62.5 \%$} \\
\hline Proportion of Level 1-Variance & \multicolumn{3}{|c|}{$37.1 \%$} & \multicolumn{3}{|c|}{$35.9 \%$} & & $7.6 \%$ & & & $37.5 \%$ & \\
\hline
\end{tabular}

Note. All estimates are linear regression coefficients based on multi-level linear regression models with vignettes (level 1) nested in respondents (level 2); $n$ (vignettes)=13,189; $n$ (respondents)=3,313. Higher values refer to more trust in the survey results.

$* \mathrm{p}<0.05 * * \mathrm{p}<0.01 * * * \mathrm{p}<0.001$.

At the same time, the results from the second model (see Column 2 in Table 2) show that survey quality indicators did play a role in the evaluation of trust in the survey result. Specifically, respondents exerted higher levels of trust with increasing sample size and when the survey was declared to be representative $(\mathrm{p}<.001 ; \mathrm{RQ} 2)$. With regard to sample size, the 
reported trust was almost half a scale point higher when the survey description stated the number of respondents to be 10,000 instead of only 100 . Interestingly, there was also a significant interaction between these two survey quality indicators $(p<.05$; see Column 4 in Table 2). According to this interaction, the effect of representativeness was most pronounced when the sample size was small (i.e., 100) while for larger sample sizes, it played only a subordinate role for the respondents whether the survey was declared as representative or not (see Figure 1). Finally, the multi-level model suggested that trust in the survey result did not change significantly, irrespective of whether a probability-based selection method was mentioned, or nothing was said about how respondents were selected $(\mathrm{p}=\mathrm{ns}$; see Column 2 in Table 2).

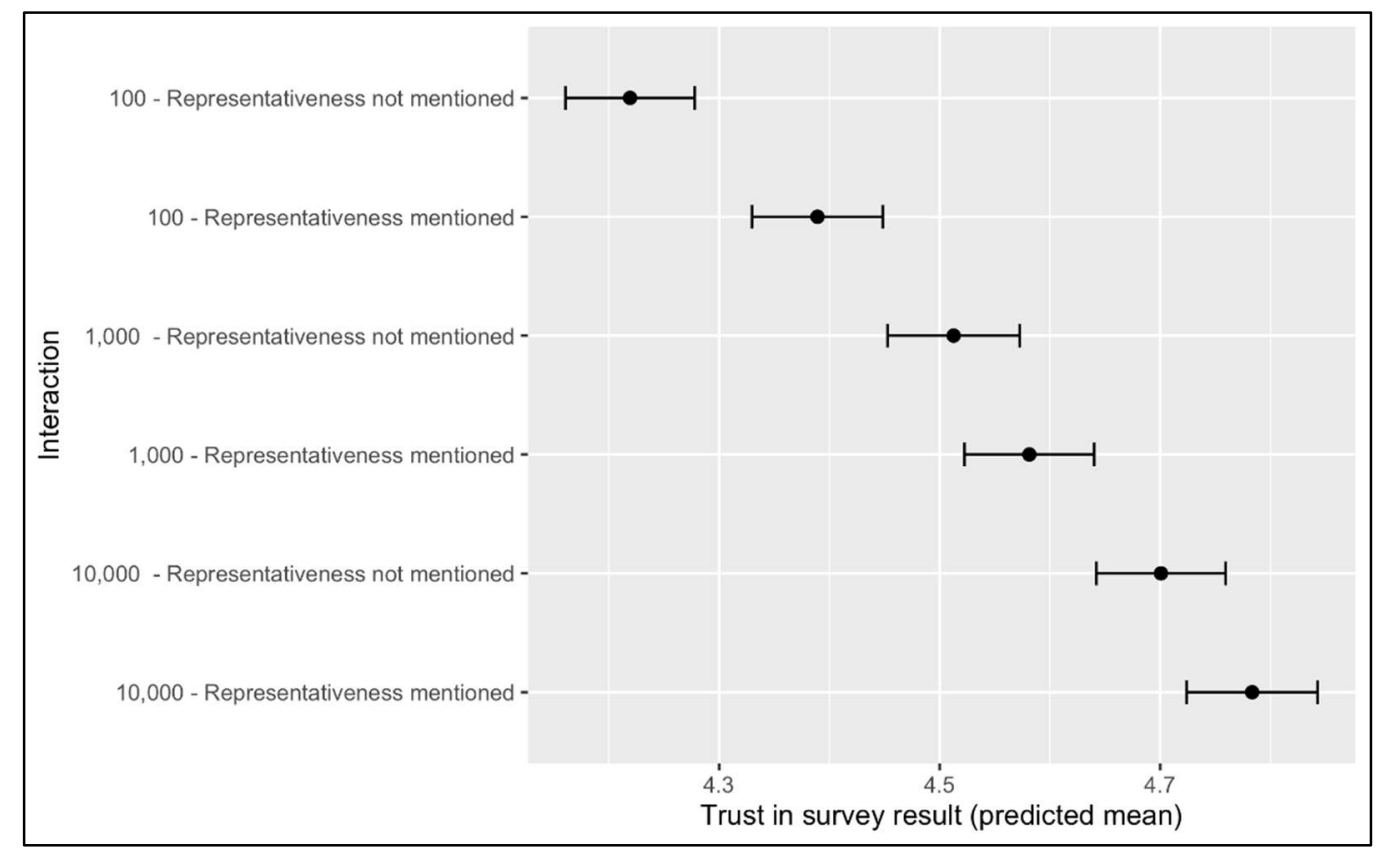

Figure 1. The combined effect of sample size and representativeness on the trustworthiness of survey results [1 "not at all" to 7 "completely"]

For the two other vignette dimensions not related to survey quality, we found a significant effect for the survey result $(\mathrm{p}<.05$; see Column 2 in Table 2), suggesting that respondents who received the vignettes with the smaller share of approval showed higher levels of trust. While there was no main effect of the source, we found an interaction effect between these two additional dimensions ( $p<.05$; see Column 4 in Table 2$)$. Accordingly, trust in the survey result was the lowest in the vignettes with the 72 percent, when this survey 
result was presented by the Automobile Association (which is the most important lobby group of drivers in Germany).

Notably, the effects of the vignette dimensions did not change when additional respondents' characteristics were included in the model (see Column 3 in Table 2). However, these control variables partly influenced the overall level of trust in the survey result. Older respondents $(p<.001)$ and respondents with higher levels of trust in science $(p<.001)$ reported more trust in the survey results.

With regard to the third research question, separate models for the three groups of formal education indicate that, as expected, people with higher education levels are more likely to rely in their assessments of trust on survey characteristics (see Table A1 to A3 in the Online Appendix). In the null models, the proportion of variance on the level of observations substantially increased from 31 (low education) to 45 percent (high education). The same holds true for the regression coefficients: For instance, the coefficient for a sample size of 10,000 was almost 4.5 times higher when the respondent reported to have a high compared to a low formal education (.814 in Column 2 in Table A1 vs. .182 in Column 2 in Tables A3). ${ }^{5}$ Accordingly, when the survey quality indicators were introduced in the model, the reduction of level 1 variance was 11.9 percent in the group of respondents with high formal education while only 1.2 percent for respondents with a low formal education. ${ }^{6}$ Finally, it is notable that the effect of the vignette dimension representativeness did not differ significantly between the three education groups (also see Table A4). This suggests that cognitive ability only functions as a moderator of specific survey quality indicators.

\footnotetext{
${ }^{5}$ A model with cross-level interactions between survey quality indicators and formal education including random slopes for the level 1 variables (Heisig and Schaeffer, 2019; see Table A4) proved that the effects of larger sample sizes on trust were significantly higher $(\mathrm{p}<0.001)$ for respondents with a high compared to respondents with a low formal education.

${ }^{6}$ This can be calculated based on the residual variances in the first and the second model. For instance, for respondents with a high formal education the reduction of level 1-variance can be calculated as follows: $((1.09-0.96) / 1.09) * 100=11.9 \%$
} 


\section{Discussion}

This paper addressed the questions on how members of the public make sense of a polling result and to what extent people rely on survey quality information when they are asked to report their perceived trust in a survey result. With regard to the first research question (RQ1), our results suggest that information on survey quality play a smaller role than characteristics of the respondents when evaluating the trustworthiness of a survey result. This holds especially true since we deliberately designed our experiment in a way that drew the participants' attention on the survey quality indicators. Given that in the news media, information on survey quality is usually placed less prominently (if at all) than in our vignettes, we may have even overestimated the relative importance of survey quality information on the trust assessments. Yet, as the results of our multi-level models indicate, the majority of the variance of the dependent variable is located on the level of the respondents, meaning that attributes of the recipients rather than attributes of the survey were more relevant when assessing trust in the survey result. By that, we extend previous research on the credibility of survey results by Kuru et al. (2017; 2020), whose findings suggested that preexisting opinions on the topic are more important than methodological descriptions. However, their design did not allow them to examine the variance components separately.

At a first glance, these results may seem discouraging for those who expected citizens to critically assess polling results when information about survey quality is at hand. However, we also found some survey quality indicators to significantly influence levels of trust (RQ2). More specifically, the results of our vignette experiment showed that the sample size but also the sample balance (i.e., the description of the survey as 'representative') received attention. Notably, the vignette experiment also indicated that some respondents in our survey seem to have regarded the sample size and the representativeness as interchangeable in the sense that a representative survey could compensate for a small sample size. 
With our study, we extended previous research, which indicated that methodological aspects can influence the credibility of a survey result (Madson and Hillygus, 2020; Salwen, 1987), by testing various methodological characteristics at once. The significant main effects of sample size and representativeness suggest that people have these survey quality indicators at hand when evaluating the trustworthiness of a survey result. To put it in the terminology of Cognitive Psychology, these two heuristics seem to be available and applicable for the general public to the domain of surveys (Tversky and Kahneman, 1974). The availability of both heuristics is not surprising. Most people should at least be able to anticipate that the reliability of a result increases with the number of people who participated in the survey. And the public supposedly became familiar with the term "representativeness" through the news media where it is a buzzword to document the methodological integrity of a survey. In contrast to previous research, the finding that mentioning that the data came from a probability-based sample increases the level of trust (Salwen, 1987) was not reproduced in our survey. This might be because our sample consisted of members of the general public, while Salwen (1987) questioned university students, who may have more knowledge about surveys. Apart from that, the expression "random selection" may possibly even evoke adverse reactions for some respondents because it may sound for them like an arbitrary process that rather indicates a survey of low methodological quality.

With respect to our third research question (RQ3) regarding a possible moderation effect by cognitive ability, the results suggest that respondents with higher levels of formal education are more likely to draw on the sample size heuristic when evaluating the trustworthiness of a survey result. More generally, our results suggest that the relative importance of survey quality information increases with the level of cognitive ability of a recipient. This finding supports information processing models of persuasion (Chaiken, 1980; Petty and Cacioppo, 1986), in which cognitive abilities are regarded as a prerequisite for message reception and comprehension. 
Future research could build on our findings in several ways. First, our study did not vary the survey topic, so that future research could test whether the results generalize to other topics. Those could be chosen in a way that respondents are confronted with issues on which they have strong and weak opinions to test whether attitude strength (e.g., How and Krosnick, 2017) moderates the effects of survey quality information. Second, qualitative or mixedmethod studies (e.g., cognitive interviews, focus groups, or web probing) may complement our findings and shed more light on the questions of how citizens make sense of polling results and of information about survey quality in particular. Such studies could also help to uncover the specific heuristics used for assessing survey quality. For example, it would be interesting to learn more about respondents' perceptions of different sampling methods or the combined effect of sample size and sampling methods.

Finally, given the increase of surveys of questionable quality, it is of growing importance to enable citizens to assess the quality of a survey and a survey result. Future research projects may thus develop educational material, conduct intervention studies, and evaluate their effects. Those efforts could be important since our findings suggest that people primarily rely on their pre-existing attitudes when evaluating the trustworthiness of a survey result, simply because this is the most available heuristic for them. The reason for the attitude driven evaluation of survey results might be that survey quality information are either not 
available (because relevant information is not reported in the media) or that many recipients are not trained to account for methodological details even if they are reported.

\section{References}

American Association of Public Opinion Research (AAPOR) (2021) The code of professional ethics and practices. Available at: www.aapor.org/Standards-Ethics/AAPOR-Code-ofEthics.aspx (accessed 31 March 2021).

Audibert C, Glass D and Johnson TP (2020) Method and transparency of online physician surveys: an overview. Survey Methods: Insights from the Field. Available at: https://surveyinsights.org/?p=12496 (accessed 31 March 2021).

Auspurg K and Hinz T (2015) Factorial Survey Experiments. Thousand Oaks: Sage.

Baker R, Blumberg SJ, Brick JM, et al. (2010) Research synthesis: AAPOR report on online panels. Public Opinion Quarterly 74(4): 711-781.

Brodie M, Parmalee L, Brackett LF, et al. (2001) Polling and democracy. Public Perspective 12(4): 10-24.

Chaiken S (1980) Heuristic versus systematic information processing and the use of source versus message cues in persuasion. Journal of Personality and Social Psychology 39(5): 752-766.

Clifford S, Jewell RM and Waggoner PD (2015) Are samples drawn from Mechanical Turk valid for research on political ideology? Research \& Politics 2(4): 1-9. 
Couper MP and Miller PV (2008) Web survey methods: Introduction. Public Opinion Quarterly 72(5): 831-835.

Gallup G and Rae SF (1940) The pulse of democracy: the public-opinion poll and how it works. Oxford: Simon \& Schuster.

Greszki R, Meyer M and Schoen H (2015) Exploring the effects of removing "too fast" responses and respondents from web surveys. Public Opinion Quarterly 79(2): 471503.

Groves RM (2011) Three eras of survey research. Public Opinion Quarterly 75(5): 861-871.

Gummer T, Roßmann J and Silber H (2018) Using instructed response items as attention checks in web surveys: Properties and implementation. Sociological Methods \& Research. Epub ahead of print 12 June 2018. DOI: 10.1177/0049124118769083.

Heisig JP and Schaeffer M (2019) Why should you always include a random slope for the lower-level variable involved in a cross-level interaction. European Sociological Review 35(2): 258-279.

Hermanni H von and Lemcke J (2017) A review of reporting standards in academic journals: A research note. Survey Methods: Insights from the Field. Available at: https://surveyinsights.org/?p=8625 (accessed 31 March 2021).

Howe LC and Krosnick JA (2017) Attitude strength. Annual review of psychology 68(1): $327-351$

Hox JJ, Moerbeek M and Van de Schoot R (2017) Multilevel Analysis: Techniques and Applications. $3^{\text {rd }}$ ed. New York: Routledge.

Kunda Z (1990) The case for motivated reasoning. Psychological bulletin 108(3): 480.

Kuru O, Pasek J and Traugott MW (2017) Motivated reasoning in the perceived credibility of public opinion polls. Public Opinion Quarterly 81(2): 422-446.

Kuru O, Pasek J and Traugott MW (2020) When polls disagree: How competitive results and methodological quality shape partisan perceptions of polls and electoral predictions. International Journal of Public Opinion Research 32(3): 586-603.

Leeper TJ (2019) Where have the respondents gone? Perhaps we ate them all. Public Opinion Quarterly 83(S1): 280-288.

Lodge M and Taber CS (2000) Three steps toward a theory of motivated political reasoning. In: Lupia A, McCubbins MD, Popkin SL (eds) Elements of reason: Cognition, choice, and the bounds of rationality. Cambridge: University Press, pp. 183-213.

Madson GJ and Hillygus DS (2020) All the best polls agree with me: Bias in evaluations of political polling. Political Behavior 42(4): 1055-1072.

Mercer AW, Kreuter F, Keeter S, et al. (2017) Theory and practice in nonprobability surveys: parallels between causal inference and survey inference. Public Opinion Quarterly 81(S1): 250-271. 
Meyer P (1990) Presidential Address: Polling as Political Science and Polling as Journalism. Public Opinion Quarterly 54(3): 451-459.

Miller MM and Hurd R (1982) Conformity to AAPOR standards in newspaper reporting of public opinion polls. Public Opinion Quarterly 46(2): 243-249.

Moy P and Rinke EM (2012) Attitudinal and behavioral consequences of published opinion polls. In: Holtz-Bacha C and Strömbäck J (eds) Opinion Polls and the Media. London: Palgrave Macmillan, pp. 225-245.

Mutz DC (2011) Population-Based Survey Experiments. Princeton: Princeton University Press.

Newport F, Shapiro RY, Ayres W, et al. (2013) Polling and democracy: Executive summary of the AAPOR task force report on public opinion and leadership. Public Opinion Quarterly 77(4): 853-860.

Petty RE and Cacioppo JT (1986) The Elaboration Likelihood Model of Persuasion. Advances in Experimental Social Psychology 19: 123-205.

Rabe-Hesketh S and Skrondal A (2012). Multilevel and Longitudinal Modeling Using Stata. $3^{\text {rd }}$ ed. College Station: StataCorp LP.

Redlawsk DP (2002) Hot cognition or cool consideration? Testing the effects of motivated reasoning on political decision making. Journal of Politics 64(4): 1021-1044.

Salwen MB (1987) Credibility of newspaper opinion polls: Source, source intent and precision. Journalism Quarterly 64(4): 813-819.

Searles K, Smith G and Sui M (2018) Partisan media, electoral predictions, and wishful thinking. Public Opinion Quarterly 82(S1): 888-910.

Sturgis P, Nick B, Mario C, et al. (2016) Report of the inquiry into the 2015 British general election opinion polls. Market Research Society and British Polling Council. Available at: http://eprints.ncrm.ac.uk/3789/ (accessed 31 March 2021).

Taber CS and Lodge M (2006) Motivated skepticism in the evaluation of political beliefs. American Journal of Political Science 50(3): 755-769.

Taber CS, Cann D and Kucsova S (2009) The motivated processing of political arguments. Political Behavior 31(2): 137-155.

Tsfati Y (2001) Why do people trust media pre-election polls? Evidence from the İsraeli 1996 elections. International Journal of Public Opinion Research 13(4): 433-441.

Tversky A and Kahneman D (1974) Judgment under uncertainty: Heuristics and biases. Science 185(4157): 1124-1131.

Verba S (1996) The citizen as respondent: sample surveys and American democracy presidential address, American Political Science Association, 1995. American Political Science Review 90(1): 1-7. 
Yeager DS, Krosnick JA, Chang L, et al. (2011) Comparing the accuracy of RDD telephone surveys and internet surveys conducted with probability and non-probability samples. Public Opinion Quarterly 75(4): 709-747. 


\section{Online Appendix}

Table A1. Results of the vignette experiment (only respondents with low education)

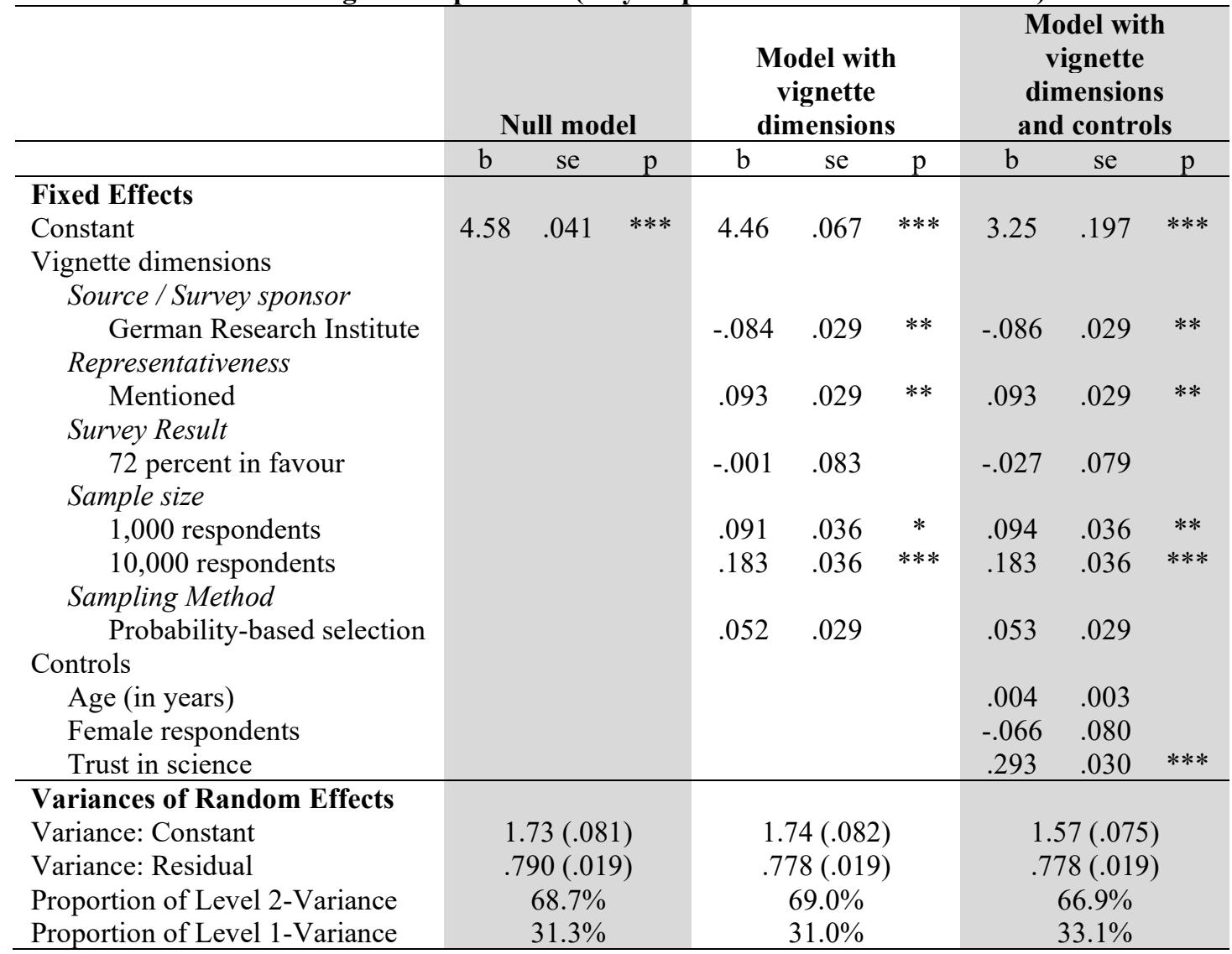

Note. All estimates are linear regression coefficients based on multi-level linear regression models with vignettes (level 1) nested in respondents (level 2); $\mathrm{n}$ (vignettes) $=4,489 ; \mathrm{n}$ (respondents) $=1,129$. Higher values refer to more trust in the survey results.

$* \mathrm{p}<0.05 * * \mathrm{p}<0.01 * * * \mathrm{p}<0.001$ 
Table A2. Results of the vignette experiment (only respondents with medium education)

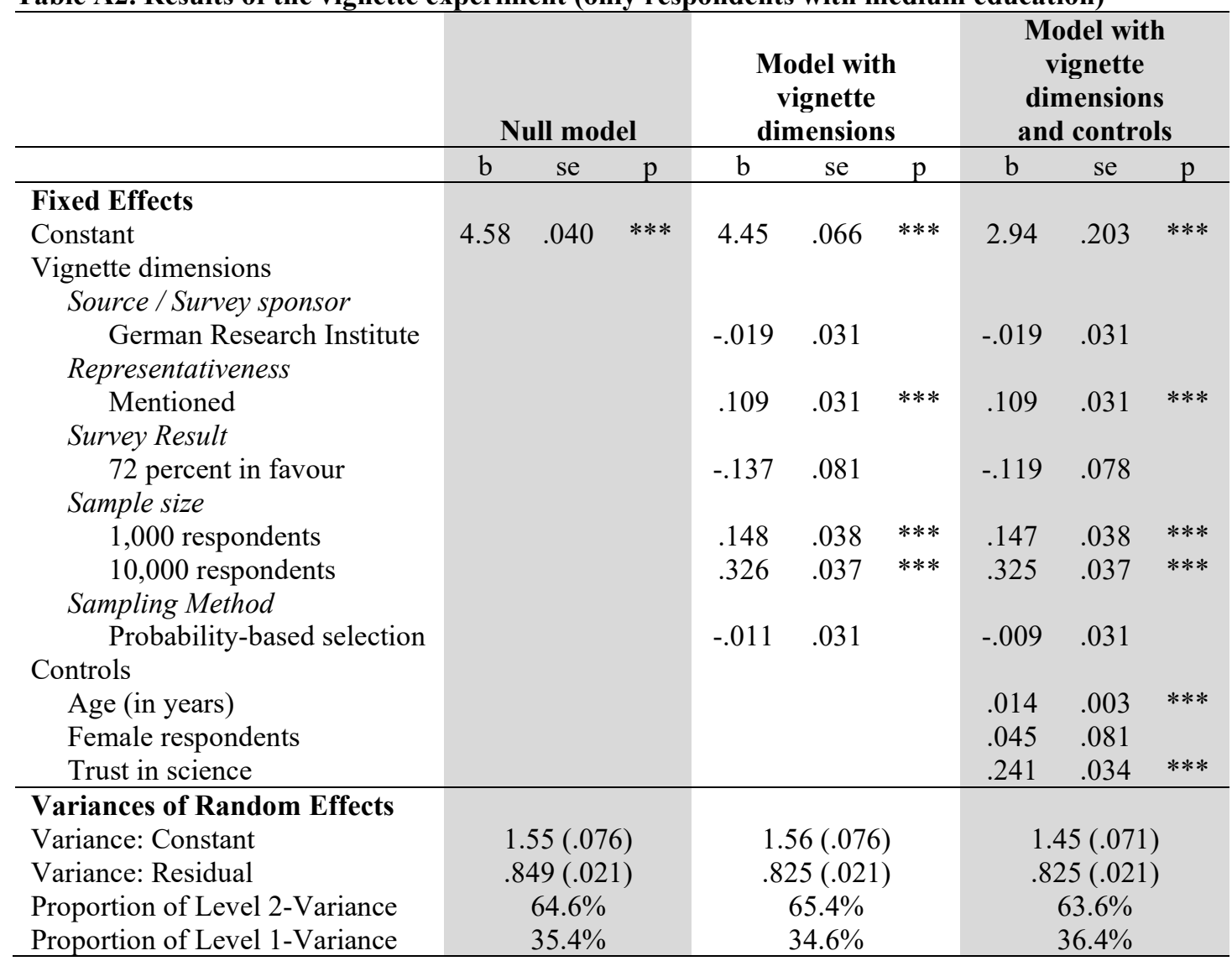

Note. All estimates are linear regression coefficients based on multi-level linear regression models with vignettes (level 1) nested in respondents (level 2); $\mathrm{n}$ (vignettes) $=4,319 ; \mathrm{n}$ (respondents) $=1,085$. Higher values refer to more trust in the survey results.

$* \mathrm{p}<0.05 * * \mathrm{p}<0.01 * * * \mathrm{p}<0.001$. 
Table A3. Results of the vignette experiment (only respondents with high education)

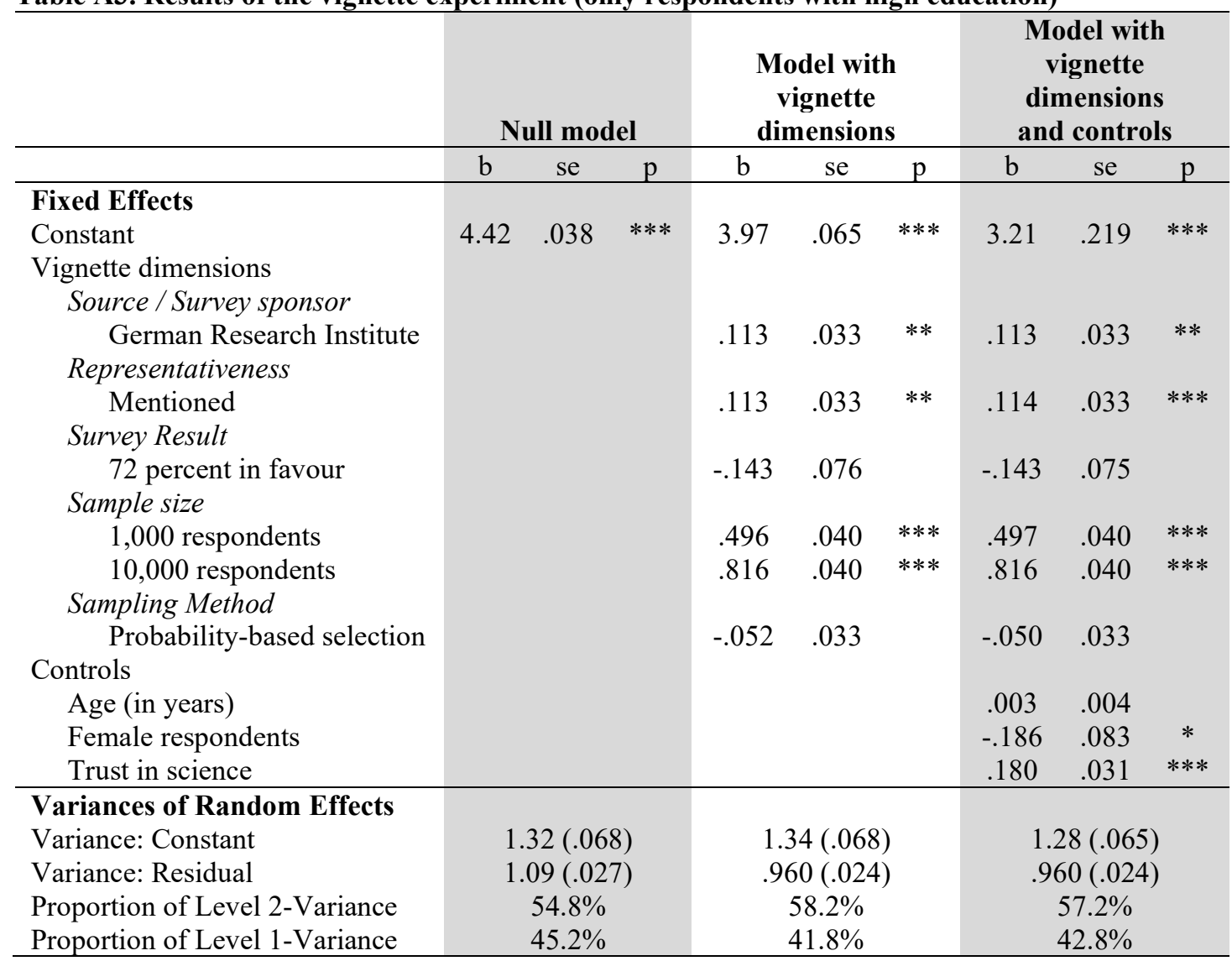

Note. All estimates are linear regression coefficients based on multi-level linear regression models with vignettes (level 1) nested in respondents (level 2); $\mathrm{n}$ (vignettes) $=4,381 ; \mathrm{n}$ (respondents) $=1,099$. Higher values refer to more trust in the survey results.

$* \mathrm{p}<0.05 * * \mathrm{p}<0.01 * * * \mathrm{p}<0.001$. 
Table A4. Results of the vignette experiment (model with cross-level interactions)

\begin{tabular}{|c|c|c|c|}
\hline & $\mathrm{b}$ & se & $\mathrm{p}$ \\
\hline \multicolumn{4}{|l|}{ Fixed Effects } \\
\hline Constant & 3.27 & .138 & $* * *$ \\
\hline \multicolumn{4}{|l|}{ Vignette dimensions } \\
\hline \multicolumn{4}{|l|}{ Source / Survey sponsor } \\
\hline German Research Institute & -.040 & .024 & \\
\hline \multicolumn{4}{|l|}{ Representativeness } \\
\hline Mentioned & .154 & .042 & $* * *$ \\
\hline \multicolumn{4}{|l|}{ Survey Result } \\
\hline 72 percent in favour & -.125 & .049 & $*$ \\
\hline \multicolumn{4}{|l|}{ Sample size } \\
\hline 1,000 respondents & .134 & .048 & $* *$ \\
\hline 10,000 respondents & .213 & .051 & $* * *$ \\
\hline \multicolumn{4}{|l|}{ Sampling Method } \\
\hline Probability-based selection & .052 & .042 & \\
\hline \multicolumn{4}{|l|}{ Formal education } \\
\hline Medium & -.027 & .072 & \\
\hline High & -.465 & .081 & $* * *$ \\
\hline \multicolumn{4}{|l|}{ Controls } \\
\hline Age (in years) & .008 & .002 & $* * *$ \\
\hline Female respondents & -.054 & .047 & \\
\hline Trust in Science & .234 & .019 & $* * *$ \\
\hline \multicolumn{4}{|l|}{ Cross-level interactions } \\
\hline Representative $\mathrm{x}$ Medium education & .016 & .043 & \\
\hline Representative $\mathrm{x}$ High education & .037 & .043 & \\
\hline 1,000 respondents $\mathrm{x}$ Medium education & .049 & .053 & \\
\hline 1,000 respondents $\times$ High education & .400 & .053 & $* * *$ \\
\hline 10,000 respondents $\mathrm{x}$ Medium education & .138 & .059 & * \\
\hline 10,000 respondents $\mathrm{x}$ High education & .629 & .058 & $* * *$ \\
\hline Probability-based x Medium education & -.066 & .043 & \\
\hline Probability-based $\mathrm{x}$ High education & -.103 & .042 & * \\
\hline \multicolumn{4}{|l|}{ Variances of Random Effects } \\
\hline Variance: Constant & \multicolumn{3}{|c|}{$1.46(.043)$} \\
\hline Variance: Representativeness & \multicolumn{3}{|c|}{$.016(.024)$} \\
\hline Variance: Sample Size & \multicolumn{3}{|c|}{$.106(.011)$} \\
\hline Variance: Sampling Method & \multicolumn{3}{|c|}{$.001(.000)$} \\
\hline Variance: Residual & \multicolumn{3}{|c|}{$.757(.015)$} \\
\hline Proportion of Level 2-Variance & \multicolumn{3}{|c|}{$65.8 \%$} \\
\hline Proportion of Level 1-Variance & \multicolumn{3}{|c|}{$34.2 \%$} \\
\hline
\end{tabular}

Note. All estimates are linear regression coefficients based on multi-level linear regression models with vignettes (level 1) nested in respondents (level 2); $n$ (vignettes) $=13,189 ; n$ (respondents) $=3,313$. Higher values refer to more trust in the survey results.

$* \mathrm{p}<0.05 * * \mathrm{p}<0.01 * * * \mathrm{p}<0.001$. 